\title{
Evidencias morfológicas y moleculares que validan como especie a Centruroides tecomanus (Scorpiones, Buthidae)
}

\section{Morphological and molecular evidence supporting specific status for Centruroides tecomanus (Scorpiones, Buthidae)}

\author{
Javier Ponce-Saavedra ${ }^{1}$, Oscar F. Francke ${ }^{2 *}$, Horacio Cano-Camacho ${ }^{3}$ y Erasto Hernández-Calderón ${ }^{1}$ \\ ${ }^{1}$ Laboratorio de Entomología “Biól. Sócrates Cisneros Paz”, Facultad de Biología, Universidad Michoacana de San Nicolás de Hidalgo, Edificio B-4, \\ $2^{\circ}$ piso. Ciudad Universitaria, 58060 Morelia, Michoacán, México. \\ ${ }^{2}$ Departamento de Zoología, Instituto de Biología, Universidad Nacional Autónoma de México. Apartado postal 70-153, 04510 México, D.F., México. \\ ${ }^{3}$ Centro Multidisciplinario de Estudios en Biotecnología (CMEB), Universidad Michoacana de San Nicolás de Hidalgo. Km. 9.5 Carretera Morelia- \\ Zinapécuaro, Posta Veterinaria. Apartado postal 53, Administración Chapultepec, 58262 Morelia, Michoacán, México. \\ *Correspondencia: offb@ibiologia.unam.mx
}

\begin{abstract}
Resumen. Se presentan evidencias morfológicas y moleculares para reconocer a Centruroides limpidus tecomanus Hoffmann (Scorpiones: Buthidae) como especie válida y no como una subespecie de Centruroides limpidus (Karsch). Se diagnostica la especie.
\end{abstract}

Palabras clave: escorpiones, taxonomía, rango específico, Centruroides limpidus, subespecies.

\begin{abstract}
Morphological and molecular evidence are provided to recognize Centruroides limpidus tecomanus Hoffmann (Scorpiones: Buthidae), as a valid species rather than a subspecies of Centruroides limpidus (Karsch). A diagnosis is provided.
\end{abstract}

Key words: scorpions, taxonomy, specific rank, Centruroides limpidus, subspecies.

\section{Introducción}

México está considerado uno de los países con mayor diversidad, ocupando el tercer sitio mundial.(LlorenteBousquets et al., 1996). La riqueza de alacranes le ubica como el país más rico con aproximadamente 216 especies descritas que representan 23 géneros pertenecientes a las 7 familias reconocidas para Norteamérica (Buthidae, Chactidae, Diplocentridae, Euscorpiidae, Iuridae, Superstitioniidae y Vaejovidae), con 17 subespecies $\mathrm{y}$ al menos 52 que para 2001 esperaban ser descritas (Beutelspacher, 2000; Fet et al., 2000, González-Santillán, 2001; González Santillán s.f.), a pesar de que hay muchas áreas geográficas del país que aún no se han estudiado. La importancia médica y biológica de estos arácnidos ha hecho que a pesar de ser un grupo relativamente pequeño, con cerca de 1450 especies descritas en el mundo (Fet et al., 2000; Rein, 2006), se le haya dedicado gran atención, debido a su importancia en el ámbito toxicológico y

Recibido: 08 noviembre 2007; aceptado: 28 julio 2008 médico; aunque en años recientes además del conocimiento sistemático, se ha incrementado el ecológico (Brown et al., 2002; Ponce, 2003; Yamashita, 2004) y los trabajos sobre la biología y comportamiento de algunas especies (Polis, 1990; Polis y Sissom, 1990; Brown, 2001; Ponce et al., 2003; Contreras-Garduño et al., 2006).

Hasta 1998, de la familia Buthidae se registraban en el mundo 73 géneros, 529 especies y 165 subespecies.(Fet et al., 2000), número que se ha incrementado con la descripción de 8 nuevos géneros y 116 especies hasta marzo del 2008 (Rein, 2006). En México, el género Centruroides Marx 1890 es el más común y diverso. Generalmente se consideraba el único representante de la familia Buthidae; sin embargo De Armas y Martín-Frías (1998) describieron Tityopsis aliciae De Armas y Martín-Frías, 1998 con un ejemplar bastante deteriorado procedente de la zona de Tehuantepec, Oaxaca. La presencia de esta especie en el país ha sido corroborada recientemente (Vidal-Acosta y Francke, en prensa). El género Centruroides contiene actualmente 65 especies y unas 10 subespecies del Nuevo Mundo. Para México se han registrado 29 especies, 5 de 
ellas con taxones subespecíficos (De Armas et al., 2003; Ponce y Moreno, 2005; Francke y Sissom, en prensa). La situación taxonómica para algunas especies de este género aún no es clara, lo que ha llevado a determinaciones erróneas y como consecuencia se han descrito patrones de distribución en los que se encuentran confundidas varias especies (Miranda, 2001; Hernández, 2002; Ponce, 2003; Ponce y Moreno, 2005).

Los alacranes como grupo se consideran animales de amplia distribución; sin embargo, en el nivel específico es relativamente común encontrar distribuciones restringidas; incluso, en escalas locales, las especies muestran patrones de distribución espacial claramente influenciados por las preferencias de microhábitat (Polis, 1990).

La distribución conocida para el género Centruroides es desde el sur de los Estados Unidos hasta Centroamérica y el norte de Sudamérica (Fet et al., 2000). En México, el género se encuentra ampliamente distribuido y contiene especies con distribuciones muy amplias como $C$. infamatus (Koch) y C. limpidus (Karsch) en el centro del país, C. gracilis (Latreille) hacia el sureste, y C. exilicauda (Wood) y C. vittatus (Say) en el norte (Beutelspacher, 2000; González-Santillán, 2001).

De acuerdo con González-Santillán (2001), en el género se reconocen 4 grupos de especies: a) grupo "rayados"que incluye las especies de Centruroides con 2 bandas longitudinales obscuras flanqueando una banda amarilla mesal que le dan la apariencia rayada; b) grupo "bertholdii", especies con 8 hileras o series de granulaciones en el borde interno de los dedos de los pedipalpos y dorsalmente sin rayas; c) grupo "gracilis", especies con 9 hileras de granulaciones en el borde interno de los pedipalpos, y d) grupo “thorelli”, especies de tamaño pequeño con pigmentación en forma de manchas sin llegar a constituir bandas y con hábitos arborícolas.

Centruroides limpidus se reconoce en la actualidad como un taxón con 2 subespecies: C. limpidus limpidus (Karsch, 1879) y C. limpidus tecomanus Hoffmann 1932. Centruroides limpidus fue descrita en 1879, con base en ejemplares provenientes del estado de Puebla (Fet et al., 2000). Posteriormente, Hoffmann (1932) describe poblaciones del estado de Colima a las cuales les asigna la categoría taxonómica de subespecie, usando como carácter diagnóstico la espina subaculear cónica y evidente tanto en hembras como en machos que contrasta con la espina subaculear poco desarrollada y roma de la subespecie nominotípica, quedando desde entonces la especie con 2 taxones subespecíficos.

La especie está ubicada en el grupo de Centruroides rayados en el que, con base en el patrón de color del caparazón cefalotorácico, se pueden distinguir 2 subgrupos: a) "limpidus-elegans", caracterizados por poseer líneas bien definidas en el carapacho y b) "suffusus-infamatus", cuyo carapacho presenta pigmentación difusa o intensa, pero sin líneas definidas (González-Santillán, 2001). El grupo de los Centruroides "rayados", tiene problemas importantes en su taxonomía debido a varios factores, como: a) la simpatría de poblaciones que al identificarlas con los criterios morfológicos actuales corresponderían a diferentes subespecies, situación que ocurre con las 2 subespecies de $C$. infamatus en Michoacán, o los registros de las 2 subespecies de $C$. limpidus en varias localidades del mismo estado (Moreno et al., 1998; Ponce y Beutelspacher, 2001; Ponce, 2003; Ponce y Francke, 2004; Ponce y Moreno, 2003, 2005) y b) la morfología tan similar entre las especies y subespecies del grupo, con simpatría de especies "crípticas", cuyas descripciones consideran una variación morfológica amplia y una distribución geográfica muy grande, provocan que con los actuales criterios para distinguir los taxones se confundan poblaciones de especies distintas en un mismo taxón.

Una situación como ésta ocurre con $C$. exilicauda (Wood) en Baja California y Sonora, donde con un análisis molecular se detectó divergencia genética entre las poblaciones de estos estados, sugiriendo 2 taxones distintos en lo que hasta ese momento se consideraba como una sola especie (Gantenbein et al., 2001), aunque aún no se ha hecho el trabajo morfológico correspondiente.

Centruroides limpidus limpidus se distribuye en los estados de Guerrero, México, Michoacán, Morelos, Querétaro y Puebla (Fet et al., 2000; González-Santillán, 2001; Córdova-Athanasiadis, 2005; Jaimes, 2007); mientras que $C$. limpidus tecomanus se registraba en simpatría con poblaciones de $C$. limpidus limpidus en diferentes localidades del estado de Michoacán, sobre todo de la depresión del Balsas (Moreno et al., 1998; Beutelspacher, 2000; Ponce y Beutelspacher, 2001), situación que posteriormente fue corregida al esclarecerse con la descripción de $C$. balsasensis Ponce y Francke 2004, taxón con el que estaba confundido. Actualmente, C. limpidus tecomanus se restringe a los estado de Colima y Michoacán, sobre la vertiente del Pacífico (Ponce, 2003; Ponce y Francke, 2004; Ponce y Moreno, 2005) y algunas localidades de Jalisco (Jaimes, 2007) (Fig. 1).

El análisis morfométrico representa una herramienta útil para el reconocimiento de diferencias entre poblaciones y la posible presencia de 2 o más taxones crípticos en una región, como muestran los trabajos que llevaron a la descripción de C. balsasensis, especie endémica de la depresión del Balsas cuya existencia se advirtió a través de la divergencia genética en las poblaciones estudiadas de Centruroides y que llevó al planteamiento de la hipótesis sobre la presencia de especies crípticas (Towler et al., 2001; Ponce y Francke, 2004). 


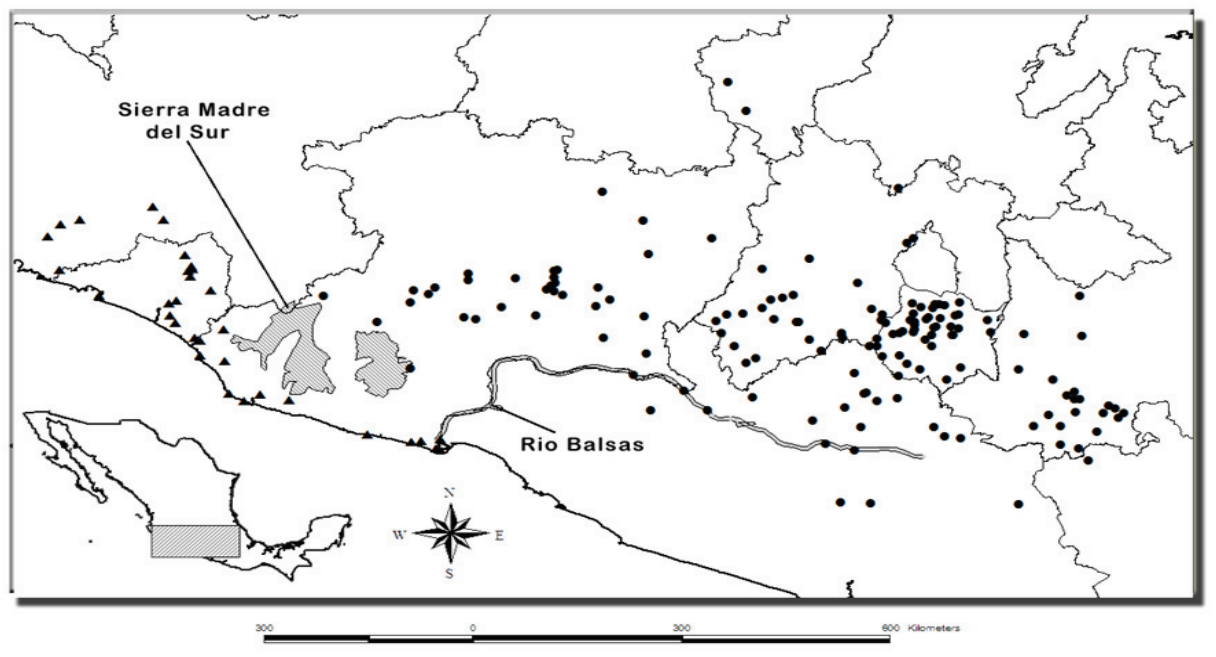

Figura 1. Distribución conocida de Centruroides limpidus limpidus (Karsch, 1879)(círculos) y de Centruroides limpidus tecomanus Hoffmann, 1932 (triángulos).

En este trabajo se presentan los resultados de análisis morfológicos y moleculares que fundamentan el cambio del estatus taxonómico subespecífico de C. limpidus tecomanus Hoffmann 1932, por el específico: C. tecomanus Hoffmann 1932.

\section{Materiales y métodos}

Análisis morfológico. Se recolectaron ejemplares en campo con ayuda de lámpara de luz negra, se preservaron en alcohol $75 \%$ y se observaron con un microscopio estereoscópico Stemi DV4. Se compararon ejemplares adultos de ambos sexos para determinar características de coloración, ornamentación y estructuras morfológicas que pudieran ser complementarias de la forma y tamaño del tubérculo subaculear que Hoffmann (1932) utilizó en la definición de las subespecies. Las mediciones se hicieron con el mismo aparato, con ayuda de un ocular micrométrico de $0.1 \mathrm{~mm}$ de precisión.

Análisis morfométrico. Se tomaron datos morfométricos de C. limpidus limpidus y C. limpidus tecomanus, y de ejemplares de $C$. balsasensis que se encuentran depositados en la colección aracnológica del Laboratorio de Entomología Biól. Sócrates Cisneros Paz, de la Facultad de Biología, Universidad Michoacana de San Nicolás de Hidalgo y de la Colección Nacional de Arácnidos del Instituto de Biología, Universidad Nacional Autónoma de México.

Se analizaron 138 individuos (69 hembras y 69 machos); la lista de ejemplares utilizados y su procedencia se muestra en el Cuadro 1. Se exploró si los individuos de las diferentes poblaciones, identificados como las subespecies en estudio, conformaban agrupaciones estadísticamente estables y se utilizó su vecino morfológicamente más parecido y geográficamente más cercano (C. balsasensis) para reconocer las variables que pueden ser definidas como diagnósticas para separar las poblaciones en el análisis.

El análisis se inició con 2 matrices, una para hembras y otra para machos, con 39 variables cada una (Cuadro 2). A estas matrices se les aplicó un análisis de correlación múltiple para identificar variables con información redundante (correlaciones de Pearson mayores de 0.9) y un análisis de componentes principales para identificar las variables informativas (mayores eigenvalores) y así eliminar ruido en los datos (Gauch, 1982; Gotelli y Ellison, 2004, Ponce, 2003; Ponce y Francke, 2004). Posteriormente se probó la capacidad individual de las variables para diferenciar los taxones y se seleccionaron las que tuvieron diferencia estadística altamente significativa $(\mathrm{p}<0.01)$, evitando al máximo seleccionar variables que midieren directamente las dimensiones de los organismos (para evitar el sesgo por tamaño) y así se obtuvieron 2 matrices, una de 9 variables para hembras y otra con 10 variables para machos. Se identificaron 7 variables que son estadísticamente distintas $(\mathrm{p}<0.01)$ entre las muestras de machos y hembras de $C$. limpidus limpidus y C. limpidus tecomanus, además de 1 variable en machos y 3 en hembras que no tuvieron diferencia estadística con los ejemplares de C. balsasensis, situación previamente establecida por Ponce y Francke (2004).

Análisis de agrupación y análisis de componentes principales $(A C P)$. Las matrices de 9 y 10 variables para hembras y machos, respectivamente, se usaron en un análisis de agrupación mediante la técnica de varianza mínima o método de Ward y el de promedios aritméticos no ponderados (UPGMA), para evaluar la estabilidad de la topología de los dendrogramas generados. Posteriormente, se aplicó un análisis de componentes principales como técnica de agrupación para reconocer las tendencias en la formación de los grupos e identificar las variables responsables. Estos análisis se hicieron con el software 
Cuadro 1. Relación de localidades y ejemplares utilizados en el análisis morfométrico

\begin{tabular}{|c|c|c|c|}
\hline Localidad & Taxón & $\begin{array}{l}\text { Núm. } \\
\text { Machos }\end{array}$ & $\begin{array}{l}\text { Núm. } \\
\text { Hembras }\end{array}$ \\
\hline $\begin{array}{l}\text { Los Mezcales, Mpio. de Comala, Colima } \\
\left(103^{\circ} 43^{\prime} 00^{\prime \prime} \mathrm{O} ; 18^{\circ} 43^{\prime} 59^{\prime \prime} \mathrm{N}\right)\end{array}$ & Centruroides limpidus tecomanus & 10 & 10 \\
\hline $\begin{array}{l}\text { Faro de Bucerias, Mpio. de Aquila, Michoacán } \\
\left(-103^{\circ} 30^{\prime} 03^{\prime \prime} \mathrm{O} ; 18^{\circ} 20^{\prime} 09^{\prime} \mathrm{N}\right)\end{array}$ & Centruroides limpidus tecomanus & 10 & 9 \\
\hline $\begin{array}{l}\text { Rancho “El Jagüey”, Mpio. de Gabriel Zamora, Michoacán } \\
\left(102^{\circ} 02^{\prime} 35^{\prime} \text { O; } 19^{\circ} 09^{\prime} 11^{\prime \prime} \mathrm{N}\right)\end{array}$ & Centruroides limpidus limpidus & 9 & 10 \\
\hline $\begin{array}{l}\text { Tzitzio, Mpio. de Tzitzio, Michoacán } \\
\left(100^{\circ} 55^{\prime} 5^{\prime \prime} \mathrm{O} ; 19^{\circ} 35^{\prime} 00^{\prime \prime} \mathrm{N}\right)\end{array}$ & Centruroides limpidus limpidus & 10 & 10 \\
\hline $\begin{array}{l}\text { Arcelia, Mpio. de Arcelia, Guerrero } \\
\left(100^{\circ} 16^{\prime} 00^{\prime \prime} \mathrm{O} ; 18^{\circ} 16^{\prime} 59^{\prime \prime} \mathrm{N}\right)\end{array}$ & Centruroides limpidus limpidus & 10 & 10 \\
\hline $\begin{array}{l}\text { Cerro de Turitzio, Mpio. de Huetamo, Michoacán }\left(100^{\circ} 55^{\prime}\right. \\
35^{\prime} \text { O O; } 18^{\circ} 32^{\prime} 25^{\prime} \text { N) }\end{array}$ & Centruroides balsasensis & 10 & 10 \\
\hline \multirow{2}{*}{$\begin{array}{l}\text { Churumuco, Mpio. de Churumuco, Michoacán } \\
\left(101^{\circ} 38^{\prime} 46^{\prime} \text { O; } 18^{\circ} 39^{\prime} 39^{\prime \prime} \mathrm{N}\right)\end{array}$} & Centruroides balsasensis & 10 & 10 \\
\hline & Total: & 69 & 69 \\
\hline
\end{tabular}

JMP versión 6.0.0 de SAS Institute (2005).

Análisis molecular. Mediante la técnica de amplificación por la reacción en cadena de la polimerasa (PCR; Simpson, 1997), se hizo amplificación de DNA y posterior secuenciación del fragmento de gen mitocondrial RNAm 16S de 21 ejemplares, 6 identificados como C. limpidus limpidus, 4 correspondientes a $C$. limpidus tecomanus, 4 de $C$. infamatus ornatus y 1 de $C$. infamatus infamatus, taxones con los que poblaciones de $C$. limpidus son parapátricas y con las que incluso se les ha registrado como simpátricas en zonas de transición del estado de Michoacán (Moreno et al., 1998), además de 6 secuencias de C. balsasensis, especie que anteriormente se confundía con estos taxones (Ponce y Francke, 2004) y que en el caso de $C$. limpidus limpidus se han registrado en simpatría (Ponce y Moreno, 2005). Las secuencias tuvieron un tamaño de aproximadamente 400 pares de bases, y se alinearon mediante el programa Clustal X (Jeannmougin et al., 1998). Para completar el análisis se usó una secuencia de $C$. exilicauda previamente registrada en Genbank (número de acceso: AJ288639) (Towler et al., 2001) y una de Centruroides nigrescens (Pocock, 1898) recolectado en la localidad de El Faro de Bucerías, municipio de Aquila, Michoacán (número de acceso: EU499947), como grupos externos. Todos los cladogramas obtenidos se enraizaron con C. nigrescens, especie del grupo de Centruroides "no rayados", para ayudar a la definición de los clados. Las localidades y números de acceso en GenBank de las 21 secuencias utilizadas en el análisis se muestran en el
Cuadro 3.

Las secuencias obtenidas se analizaron primero mediante el método de reconstrucción filogenético del "vecino más cercano" (neighbor-joining) que es la versión simplificada del método de mínima evolución (ME) en el que se genera un solo árbol final y posteriormente aplicar el algoritmo de ME para probar la consistencia de los clados obtenidos. El método de remuestreo o bootstraping (con 500 réplicas) se aplicó para estimar el grado de soporte de los clados. Los parámetros utilizados en el software fueron: número máximo de árboles retenidos $=1$; inicio del procedimiento con un árbol generado por el método de vecino más cercano; con 1 CNI (close-neighborinterchange heuristic search) recomendable por el número de secuencias utilizadas (> 15) y usando el modelo de nucleótidos de Kimura-2, el cual admite igualdad en la frecuencia de nucleótidos y considera tanto la tasa de transición como la de transversión. Para el análisis se utilizó el software MEGA (Molecular Evolutionary Genetics Analysis) versión 3.1 (Kumar et al., 2004).

\section{Redescripción}

Centruroides tecomanus Hoffmann 1932 stat. nov.

Sinonimia (hasta el 31 de diciembre de 1998 ver Fet et al., 2000).

Centruroides limpidus tecomanus: Fet et al. 2000: 112; 
Cuadro 2. Variables utilizadas inicialmente en la matriz para la clasificación de los machos y hembras de los 3 taxones del grupo "limpidus" representados en el análisis $(\mathrm{n}=39)$

\begin{tabular}{|c|c|c|}
\hline Medidas & Conteos & Proporciones \\
\hline Longitud total & Número de dientes pectinales $*$ & Longitud total /long. del metasoma \\
\hline Longitud del cefalotórax & Dientes accesorios externos del dedo móvil & Longitud del metasoma/long. del cefalotórax \\
\hline Ancho del cefalotórax & Dientes accesorios internos del dedo móvil & Longitud del cefalotórax/long. del segmento caudal V \\
\hline Longitud del mesosoma & Dientes accesorios externos del dedo fijo & Longitud del cefalotórax/long. del dedo móvil \\
\hline Longitud del metasoma & Dientes accesorios internos del dedo fijo & Proporción longitud/ancho de la patela \\
\hline Longitud de la mano & Tamaño y forma del tubérculo subaculear ** & Longitud del dedo fijo/long. del dedo móvil \\
\hline Longitud del dedo móvil & & Proporción longitud/ancho del segmento caudal V \\
\hline Longitud del dedo fijo & & Longitud de la vesícula/ancho de la vesícula \\
\hline Longitud del fémur & & Longitud de la vesícula/long. del aguijón \\
\hline \multicolumn{3}{|l|}{ Longitud de la patela } \\
\hline \multicolumn{3}{|l|}{ Ancho de la patela } \\
\hline \multicolumn{3}{|l|}{$\begin{array}{l}\text { Longitud del segmento } \\
\text { caudal I }\end{array}$} \\
\hline \multicolumn{3}{|l|}{$\begin{array}{l}\text { Ancho del segmento } \\
\text { caudal I }\end{array}$} \\
\hline \multicolumn{3}{|l|}{$\begin{array}{l}\text { Longitud del segmento } \\
\text { caudal II }\end{array}$} \\
\hline \multicolumn{3}{|l|}{$\begin{array}{l}\text { Ancho del segmento } \\
\text { caudal II }\end{array}$} \\
\hline \multicolumn{3}{|l|}{$\begin{array}{l}\text { Longitud del segmento } \\
\text { caudal III }\end{array}$} \\
\hline \multicolumn{3}{|l|}{$\begin{array}{l}\text { Ancho del segmento } \\
\text { caudal III }\end{array}$} \\
\hline \multicolumn{3}{|l|}{$\begin{array}{l}\text { Longitud del segmento } \\
\text { caudal IV }\end{array}$} \\
\hline \multicolumn{3}{|l|}{$\begin{array}{l}\text { Ancho del segmento } \\
\text { caudal IV }\end{array}$} \\
\hline \multicolumn{3}{|l|}{$\begin{array}{l}\text { Longitud del segmento } \\
\text { caudal V }\end{array}$} \\
\hline \multicolumn{3}{|l|}{$\begin{array}{l}\text { Ancho del segmento } \\
\text { caudal V }\end{array}$} \\
\hline \multicolumn{3}{|l|}{ Longitud de la vesícula } \\
\hline \multicolumn{3}{|l|}{ Ancho de la vesícula } \\
\hline Longitud del aguijón & & \\
\hline
\end{tabular}

*Dato promedio de ambos peines.

**Variable calificada por apreciación. 0 = gránulo poco aparente, 1 = gránulo aparente, 2 = diente cónico pequeño, 3 = diente cónico mediano, 4 = diente cónico grande a muy grande.

Beutelspacher, 2000: 127; González Santillán, 2001: 105; Lourenço y Sissom, 2000: 133; Ponce y Beutelspacher, 2001: 63; De Armas et al., 2003: 94; Ponce, 2003: 111; Ponce y Francke, 2004: 221; Ponce y Moreno, 2005: 45, 50; Jaimes, 2007: 35.

Tipos. Según el catalogo elaborado por Fet y Lowe (2000), con respecto a Centruroides limpidus tecomanus Hoffmann indican: "SYNTYPES: $\widehat{T} 0$ (IBUNM), Colima, Mexico; 14仓̂, $19+$ (IBUNM), Manzanillo, Tecomán and
Colima, Colima, Mexico".

Sin embargo, existe un holotipo hembra designado por Hoffmann, depositado en el American Museum of Natural History, Nueva York (Lauren Esposito y Lorenzo Prendini, com. pers.).

Diagnosis. Alacranes de tamaño mediano (longitud total machos $67.2 \pm 8.4 \mathrm{~mm}$; hembras $63.4 \pm 5.8 \mathrm{~mm}$ ); alargados y con los pedipalpos y quelas delgadas, de color amarillo a ocre. Caparazón cefalotorácico con 4 líneas bien 
Cuadro 3. Procedencia, taxón, número de ejemplares y número de acceso en GenBank de las secuencias del fragmento de gen mitocondrial RNAm 16S utilizadas en el análisis

\begin{tabular}{|c|c|c|c|c|}
\hline Procedencia (Localidad y/o Municipio) & $\begin{array}{l}\text { Coordenadas } \\
\text { geográficas }\end{array}$ & Taxón & $\begin{array}{l}\text { Núm. } \\
\text { Sec. }\end{array}$ & $\begin{array}{l}\text { Número de acceso en } \\
\text { Genbank }\end{array}$ \\
\hline Morelia, Mpio. de Morelia, Michoacán & $\begin{array}{l}101^{\circ} 11^{\prime} 03^{\prime \prime} \mathrm{O} \\
19^{\circ} 42^{\prime} 00^{\prime \prime} \mathrm{N}\end{array}$ & C. infamatus ornatus & 1 & $\operatorname{AF} 439756(410 \mathrm{pb}) *$ \\
\hline Tiripetío, Mpio. de Morelia, Michoacán & $\begin{array}{l}101^{\circ} 20^{\prime} 52^{\prime \prime} \mathrm{O} \\
19^{\circ} 32^{\prime} 43^{\prime \prime} \mathrm{N}\end{array}$ & C. infamatus ornatus & 1 & $\mathrm{AF} 439757(411 \mathrm{pb}) * *$ \\
\hline La Caratacua, Mpio. de Coeneo, Michoacán & $\begin{array}{l}101^{\circ} 43^{\prime} 05^{\prime \prime} \mathrm{O} \\
19^{\circ} 45^{\prime} 37^{\prime \prime} \mathrm{N}\end{array}$ & C. infamatus ornatus & 1 & $\mathrm{AF} 439755(409 \mathrm{pb}) *$ \\
\hline El Cóbano, Mpio. de Gabriel Zamora, Michoacán & $\begin{array}{l}102^{\circ} 04^{\prime} 20^{\prime \prime} \mathrm{O} \\
19^{\circ} 16^{\prime} 20^{\prime \prime} \mathrm{N}\end{array}$ & C. infamatus ornatus & 1 & AF439754 (408 pb)* \\
\hline Zumpimito, Mpio. de Uruapan, Michoacán & $\begin{array}{l}102^{\circ} 04^{\prime} 13^{\prime \prime} \mathrm{O} \\
19^{\circ} 21^{\prime} 25^{\prime \prime} \mathrm{N}\end{array}$ & C. infamatus infamatus & 1 & $\operatorname{AF} 439753(413 \mathrm{pb}) *$ \\
\hline Huitzuco, Mpio. de Huitzuco, Guerrero & $\begin{array}{l}99^{\circ} 20^{\prime} 05^{\prime \prime} \mathrm{O} \\
18^{\circ} 18^{\prime} 19^{\prime \prime} \mathrm{N}\end{array}$ & C. limpidus limpidus & 2 & $\begin{array}{l}\text { AF439763 }(411 \mathrm{pb}) * \\
\text { EU499940 }(390 \mathrm{pb})\end{array}$ \\
\hline Tacámbaro, Mpio. de Tacámbaro, Michoacán & $\begin{array}{l}101^{\circ} 27^{\prime} 04^{\prime \prime} \mathrm{O} \\
19^{\circ} 14^{\prime} 00^{\prime \prime} \mathrm{N}\end{array}$ & C. limpidus limpidus & 1 & EU492908 (391 pb) \\
\hline Tztzio, Mpio. de Tzitzio, Michoacán & $\begin{array}{l}100^{\circ} 55^{\prime} 05^{\prime \prime} \mathrm{O} \\
19^{\circ} 35^{\prime} 00^{\prime \prime} \mathrm{N}\end{array}$ & C. limpidus limpidus & 1 & EU499938 (355 pb) \\
\hline $\begin{array}{l}\text { Los Plátanos, Mpio. de Santo Tomás, Estado de } \\
\text { México. }\end{array}$ & $\begin{array}{l}100^{\circ} 13^{\prime} 30^{\prime \prime} \mathrm{O} \\
19^{\circ} 09^{\prime} 21^{\prime \prime} \mathrm{N}\end{array}$ & C. limpidus limpidus & 1 & EU499939 (377 pb) \\
\hline $\begin{array}{l}\text { Cañón de los Cajones, Mpio. de Santiago de } \\
\text { Querétaro, Querétaro }\end{array}$ & $\begin{array}{l}100^{\circ} 25^{\prime} 39^{\prime \prime} \mathrm{O} \\
20^{\circ} 40^{\prime} 47^{\prime} \mathrm{N}\end{array}$ & C. limpidus limpidus & 1 & AF439764 (408 pb)* \\
\hline El Faro, Mpio. de Aquila, Michoacán & $\begin{array}{l}103^{\circ} 30^{\prime} 03^{\prime \prime} \mathrm{O} \\
18^{\circ} 20^{\prime} 09^{\prime \prime} \mathrm{N}\end{array}$ & C. limpidus tecomanus & 3 & $\begin{array}{l}\text { AF439765 (408 pb)* } \\
\text { AF439766 (411 pb)* } \\
\text { EU499933 (388 pb) }\end{array}$ \\
\hline $\begin{array}{l}\text { Barra de Piche, Mpio. de Lázaro Cárdenas, } \\
\text { Michoacán }\end{array}$ & $\begin{array}{l}102^{\circ} 19^{\prime} 37^{\prime}, \mathrm{O} \\
17^{\circ} 58^{\prime} 24^{\prime} \mathrm{N}\end{array}$ & C. limpidus tecomanus & 1 & EU949935 (385 pb) \\
\hline Tzirándaro, Mpio. de Tzirándaro, Guerrero & $\begin{array}{l}100^{\circ} 58^{\prime} 46^{\prime \prime} \mathrm{O} \\
18^{\circ} 28^{\prime} 37^{\prime} \mathrm{N}\end{array}$ & C. balsasensis & 1 & EU499934 (388 pb) \\
\hline Churumuco, Mpio. de Churumuco, Michoacán & $\begin{array}{l}101^{\circ} 38^{\prime} 46^{\prime \prime} \mathrm{O} \\
18^{\circ} 39^{\prime} 39^{\prime \prime} \mathrm{N}\end{array}$ & C. balsasensis & 2 & $\begin{array}{l}\operatorname{AF} 439759(411 \mathrm{pb}) * * * \\
\text { EU499936 (373 pb) }\end{array}$ \\
\hline $\begin{array}{l}\text { Cerro de Turitzio (Arúa), Mpio. de Huetamo, } \\
\text { Michoacán }\end{array}$ & $\begin{array}{l}100^{\circ} 55^{\prime} 05^{\prime}, \mathrm{O} \\
19^{\circ} 35^{\prime} 00^{\prime \prime} \mathrm{N}\end{array}$ & C. balsasensis & 2 & $\begin{array}{l}\text { AF439760 }(417 \mathrm{pb}) * * * \\
\text { EU499937 }(395 \mathrm{pb})\end{array}$ \\
\hline Tzitzio, Mpio. de Tzitzio, Michoacán & $\begin{array}{l}100^{\circ} 55^{\prime} 5^{\prime \prime}, \mathrm{O} \\
19^{\circ} 35^{\prime} 00^{\prime \prime} \mathrm{N}\end{array}$ & C. limpidus limpidus & 1 & AF439762 (405 pb) *** \\
\hline
\end{tabular}

* Secuencias utilizadas en Towler et al. (2001).

** Secuencia registrada como Centruroides infamatus infamatus por Towler et al. (2001) y cuya identificación correcta es Centruroides infamatus ornatus.

*** Secuencias registradas por Towler et al. (2001) como Centruroides limpidus limpidus y cuya identificación correcta es Centruroides balsasensis.

definidas, las 2 medias sin llegar al borde posterior, lo que le ubicaría como un Centruroides "rayado". Mesosoma con 2 bandas longitudinales obscuras a los lados de una banda mesal de color claro. Las bandas obscuras están formadas por manchas sobre el preterguito y el posterguito de cada uno de los segmentos mesosomales, la mancha del posterguito con forma triangular. La coloración varía con las poblaciones, pero es común encontrar en C. tecomanus pigmentación obscura en los extremos de los segmentos caudales, fémur, patela y quelas, contrastando con el color claro que en general tienen estos animales. La diferencia morfológica más evidente con $C$. limpidus, además del tamaño y la proporción del metasoma respecto a la longitud total, se encuentra en el tubérculo subaculear, el cual en C. tecomanus está bien desarrollado, es cónico y la punta se encuentra orientada hacia la punta del aguijón (Fig. 8). Adicionalmente hay diferencias en la distribución geográfica ya que $C$. tecomanus se encuentra en la región costera del Pacífico de México, desde el sur del estado de Michoacán en los límites con el estado de Guerrero hasta el 
Cuadro 4. Variables que tuvieron diferencia estadística significativa en la comparación de Centruroides limpidus limpidus y C. limpidus tecomanus. La comparación se hizo con prueba de $t$ para las medidas y Ji-cuadrada $\left(\mathrm{X}^{2}\right)$ para proporciones y conteos

\begin{tabular}{|c|c|c|c|c|}
\hline $\begin{array}{l}\text { Machos } \\
\text { Variable }\end{array}$ & $\begin{array}{c}\text { Media ( } \pm \text { D.S.) } \\
\text { C. limpidus limpidus }\end{array}$ & $\begin{array}{c}\text { Media }( \pm \text { D.S.) } \\
\text { C. limpidus tecomanus }\end{array}$ & Prob. & $S$ \\
\hline Long. total/long. metasoma & $1.51 \pm 0.04$ & $1.48 \pm 0.03$ & $<0.0001$ & alto \\
\hline Long. metasoma/long. cefalotórax & $7.31 \pm 0.18$ & $7.69 \pm 0.25$ & $<0.0001$ & alto \\
\hline Long. cefalotórax/long. segmento caudal V & $0.69 \pm 0.02$ & $0.67 \pm 0.02$ & $<0.001$ & alto \\
\hline Long. diente. móvil/long. patela & $1.03 \pm 0.03$ & $1.00 \pm 0.04$ & 0.0023 & alto \\
\hline Longitud del aguijón (mm) & $2.81 \pm 0.27$ & $3.14 \pm 0.25$ & $<0.0001$ & alto \\
\hline Dientes accs. externos (diente. fijo) & $33.34 \pm 2.69$ & $35.6 \pm 3.22$ & 0.0006 & alto \\
\hline Tubérculo subaculear* & $1.03 \pm 0.13$ & $3.85 \pm 0.37$ & $<0.0001$ & alto \\
\hline Hembras & Media $( \pm D . S)$. & Media $( \pm$ D.S. $)$ & & \\
\hline Variable & C. limpidus limpidus & C. limpidus tecomanus & Prob. & $S$ \\
\hline Long. total/long. metasoma & $1.65 \pm 0.04$ & $1.60 \pm 0.04$ & $<0.0001$ & alto \\
\hline Long. metasoma/long. cefalotórax & $6.04 \pm 0.27$ & $6.41 \pm 0.24$ & $<0.0001$ & alto \\
\hline Long. Cefalotórax/long. segmento caudal V & $0.84 \pm 0.02$ & $0.80 \pm 0.02$ & $<0.0001$ & alto \\
\hline Long. dedo fijo (mm) & $5.68 \pm 0.48$ & $6.17 \pm 0.53$ & $<0.0001$ & alto \\
\hline Ancho segmento caudal III (mm) & $2.91 \pm 0.24$ & $3.37 \pm 0.32$ & $<0.0001$ & alto \\
\hline Ancho segmento caudal IV (mm) & $2.83 \pm 0.27$ & $3.33 \pm 0.29$ & $<0.0001$ & alto \\
\hline Tubérculo subaculear* & $1.63 \pm 0.14$ & $4.00 \pm 0.18$ & $<0.0001$ & alto \\
\hline
\end{tabular}

*Variable calificada por apreciación. 0 = gránulo poco aparente, 1 = gránulo aparente, 2 = diente cónico pequeño, $3=$ diente cónico mediano, 4 = diente cónico grande a muy grande. Prob. = probabilidad; $S=$ nivel de significancia (Fig.8).

sur del estado de Jalisco, con varias localidades registradas en el estado de Colima; mientras que C. limpidus es una especie continental con registros en el estado de México, Puebla, Guerrero y Querétaro además del estado de Michoacán, siempre en localidades con climas cálidos y secos; mientras que $C$. tecomanus al ser habitante de regiones costeras, los climas son cálidos y húmedos, con las diferencias de vegetación que corresponden a estas condiciones climáticas. Además de encontrarse en suelo y rocas, $C$. tecomanus puede encontrarse por las noches en los troncos y ramas de las palmeras u otros árboles llegando a alturas de hasta $10 \mathrm{~m}$ (observación personal del primer autor); mientras que $C$. limpidus es habitante del suelo y rocas; en árboles se les encuentra sólo bajo cortezas secas durante el día. Otras diferencias son morfométricas y se presentan en el Cuadro 4.

Análisis morfométrico. Métodos de agrupación. La agrupación obtenida para los machos de las poblaciones analizadas, permite identificar 3 poblaciones morfométricamente diferentes: una que corresponde a C. limpidus tecomanus, de las localidades de El Faro de
Bucerías, municipio de Aquila en Michoacán y los Mezcales en el estado de Colima; otra para ejemplares de C. limpidus limpidus, de Tiztzio y Gabriel Zamora en Michoacán y de Arcelia en Guerrero; y una tercera población que agrupó los ejemplares de las localidades de la depresión del Balsas y que corresponden a C. balsasensis. Esta especie se separa claramente de los $C$. limpidus limpidus y los C. limpidus tecomanus en los machos; sin embargo, aún se observa un ejemplar de $C$. limpidus tecomanus que se confunde con C. balsasensis (Fig. 2); mientras que en las hembras, el grupo de C. balsasensis es muy consistente (Fig. 3). El alto porcentaje de buena clasificación (98\%) fue probado con un análisis de la función discriminante, lo que permite sugerir como útil el conjunto de características utilizadas para separar estos 3 taxones (Cuadro 4).

Análisis de componentes principales. La ordenación obtenida con el método de análisis de componentes principales (ACP) mostró una clasificación consistente (Figs. 4 y 5) con la información obtenida con los métodos de agrupación y permitió definir las 7 características que separan los C. limpidus limpidus de C. limpidus tecomanus, 


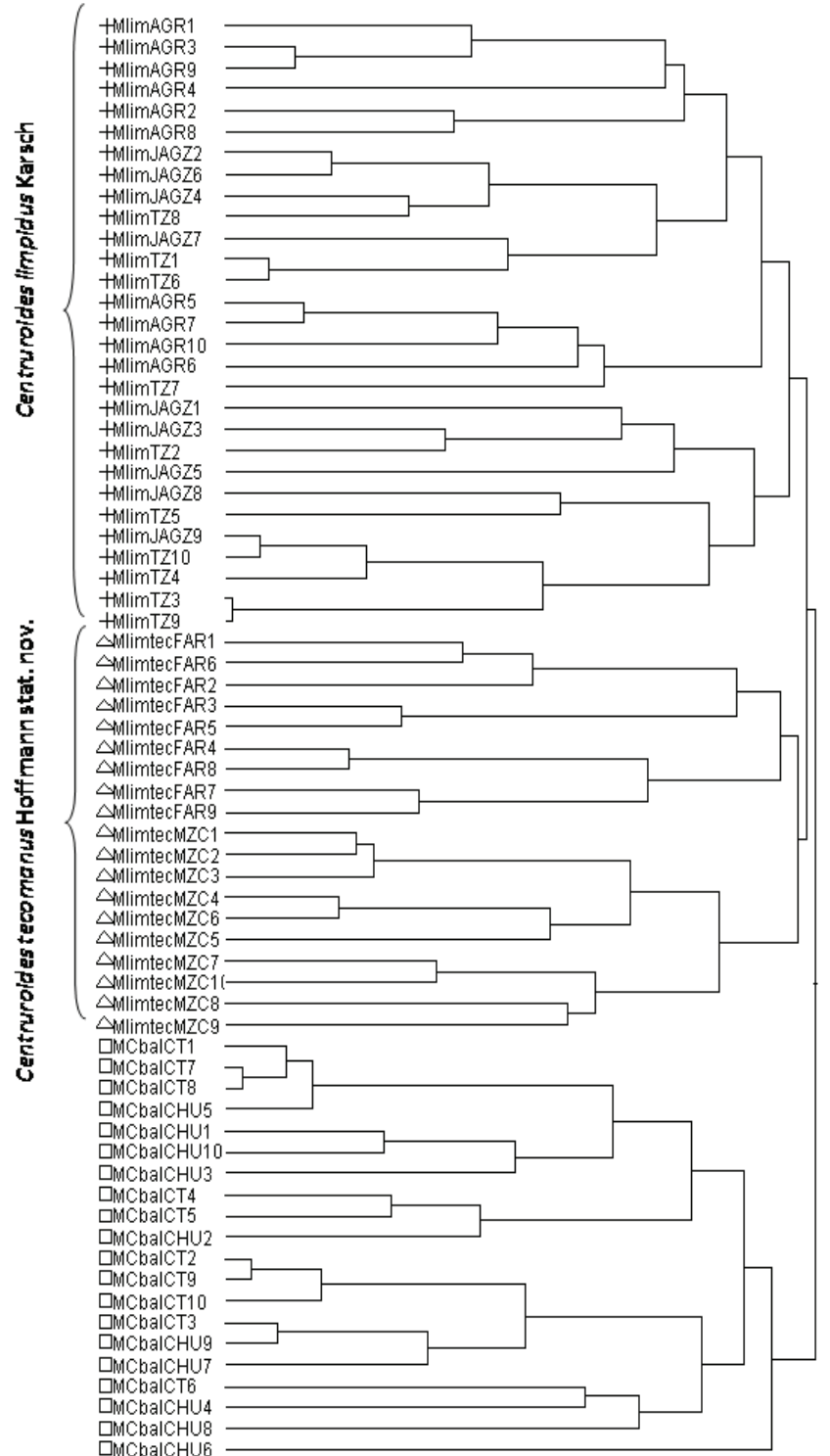

Figura 2. Dendrograma (método de Ward y distancias euclidianas) para 10 variables morfométricas utilizadas para distinguir los machos de los 3 taxones analizados.

Las abreviaturas representan la primera determinación y procedencia de los ejemplares utilizados. Para Centruroides limpidus: MlimAGRO= Arcelia, Guerrero; MlimJAGZ= Rancho "El Jaguey", Gabriel Zamora, Michoacán; MlimTZ= Tzitzio, Michoacán. Para Centruroides limpidus tecomanus: MlimtecFAR= "El Faro", Aquila, Michoacán; MlimtecMZC= "Los Mezcales", Comala, Colima. Para Centruroides balsasensis: MCbalCT $=$ Cerro de Turitzio, Arúa, Huetamo, Michoacán; MCbalCHU = Churumuco, Michoacán.

tanto en hembras como en machos, las cuales se probaron individualmente mediante una prueba de $t$ para las medidas y $X^{2}$ para las proporciones y conteos (Cuadro 4). Análisis molecular. Análisis de secuencias (RNAm 16S).

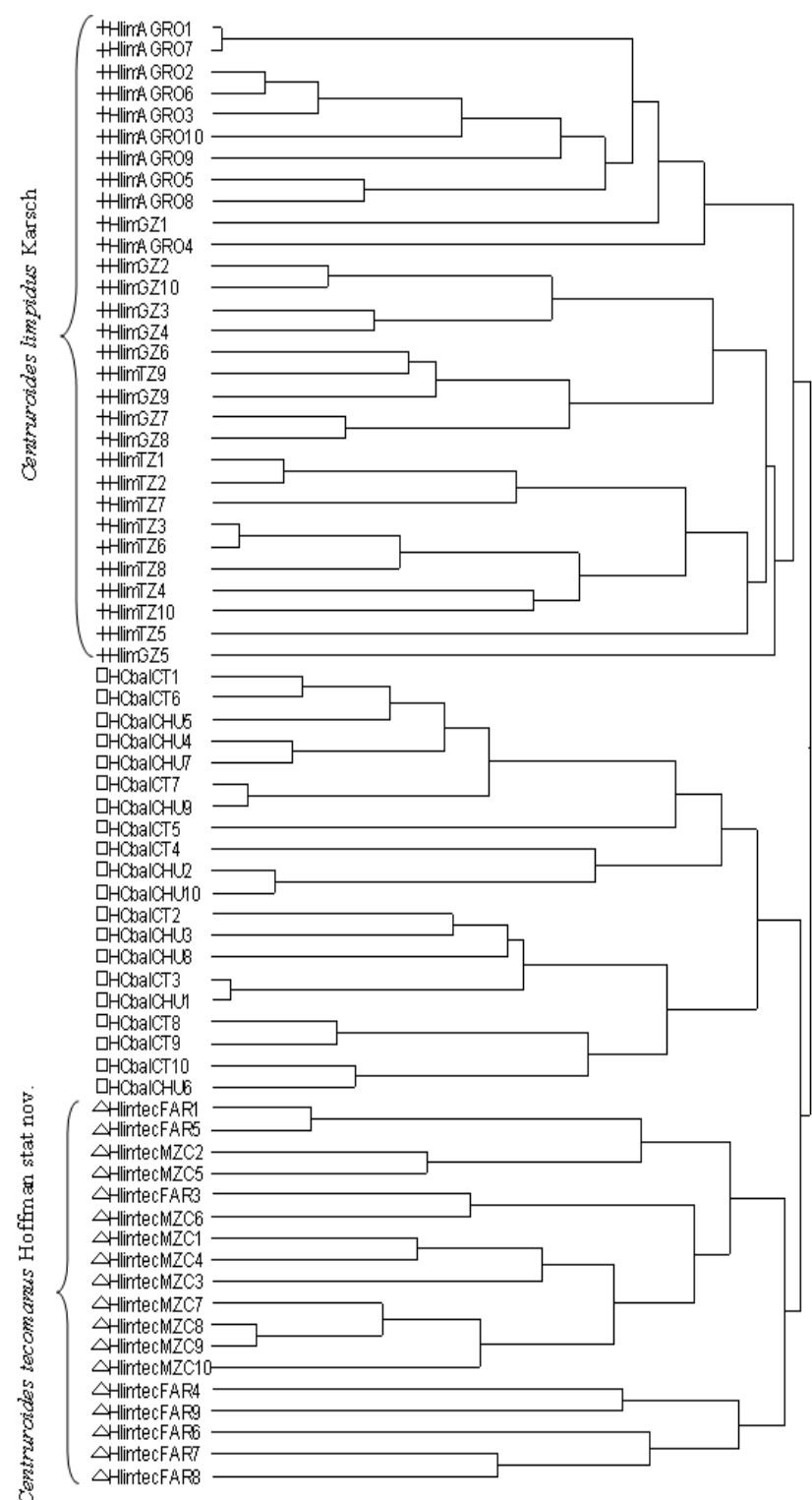

Figura 3. Dendrograma (método de Ward y distancias euclidianas) para 9 variables morfométricas utilizadas para distinguir las hembras de los 3 taxones analizados.

Las abreviaturas representan la primera determinación y procedencia de los ejemplares utilizados. Para Centruroides limpidus: HlimAGRO= Arcelia, Guerrero; HlimGZ= Rancho "El Jaguey", Gabriel Zamora, Michoacán; HlimTZ= Tzitzio, Michoacán.Para Centruroides limpidustecomanus: HlimtecFAR= "El Faro", Aquila, Michoacán; HlimtecMZC= "Los Mezcales", Comala, Colima. Para Centruroides balsasensis: HCbalCT= Cerro de Turitzio, Arúa, Huetamo, Michoacán; HCbalCHU= Churumuco, Michoacán.

Trabajando con las secuencias del fragmento de gen ribosomal $16 \mathrm{~S}$, la divergencia obtenida es clara al utilizar un método de análisis de semejanza simple, como el de vecino más cercano (Fig. 6), o bien, cuando se utilizó un 


\begin{tabular}{|c|c|c|c|}
\hline & CP1 & CP2 & CP3 \\
\hline Eigenvalor & 3.676 & 2.0741 & 1.1094 \\
\hline Poncentaie & 40.8446 & 23.0454 & 12.3267 \\
\hline \% acamilado & 40.8446 & 63.89 & 76.2167 \\
\hline \multicolumn{4}{|l|}{ Calficaciones } \\
\hline Conteopectinal & -0.07955 & 0.49599 & 0.03473 \\
\hline Long totahlong. metasoma & -0.36597 & 0.01785 & 0.2837 \\
\hline Long metal Long cefalotórax & 0.33956 & -0.33541 & 0.27991 \\
\hline $\begin{array}{l}\text { Long. cefalotóraxhlong. } \\
\text { segmento Caudal V }\end{array}$ & -0.31662 & 0.46407 & -0.21944 \\
\hline Longind /Ancho patela & -0.08032 & 0.11593 & 0.87188 \\
\hline Long̣iud agyö́n & 0.44112 & 0.32584 & -0.01035 \\
\hline $\begin{array}{l}\text { Dientes accesorios externos del } \\
\text { dedo fio }\end{array}$ & 0.4 & 0.2 & -0.02078 \\
\hline Tubéncilo subacalear (0-4) & 0.29394 & -0.31997 & -0.10144 \\
\hline
\end{tabular}

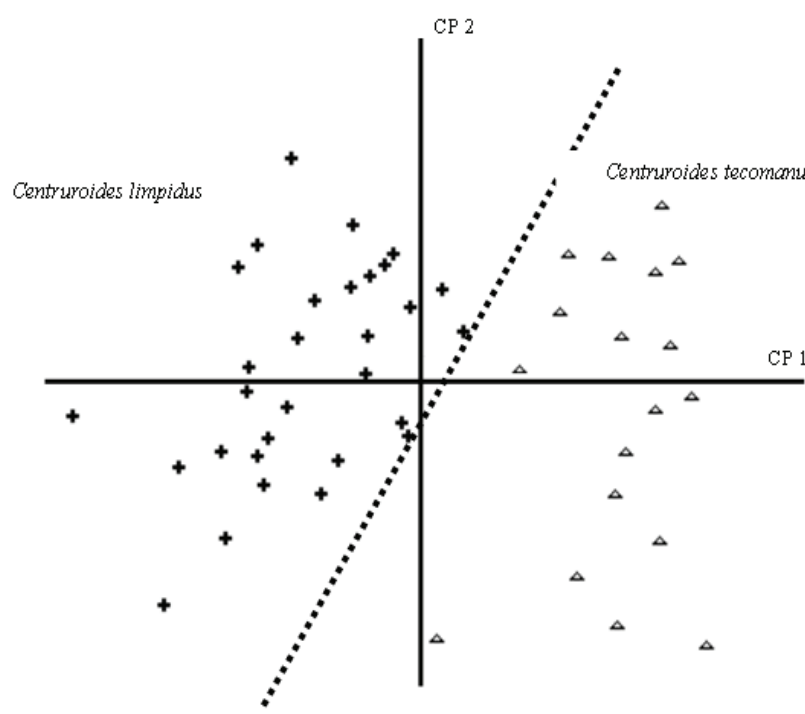

Figura 4. Gráfico de ordenación con ACP y la tabla correspondiente para 9 variables de hembras provenientes de poblaciones identificadas como subespecies de Centruroides limpidus, separadas por la línea punteada. En la tabla se resaltan las calificaciones que permiten interpretar el gráfico. Las carácterísticas en negritas del CP1 deben considerarse diagnósticas.

algoritmo más complejo, como el de mínima evolución (Fig. 7). Los árboles obtenidos con ambos métodos son idénticos en su topología, apreciándose que la polarización hecha con $C$. nigrescens es buena y corrobora que este representante de los Centruroides "no rayados", tiene diferencias genéticas suficientes respecto a los taxones de Centruroides "rayados" utilizados en este análisis, al identificarse un clado con linajes que agrupan poblaciones de C. limpidus limpidus claramente separado de un clado que contiene las poblaciones de C. limpidus tecomanus, el cual tiene mayor proximidad genética con el clado formado por los ejemplares representantes de las poblaciones de $C$. infamatus; con el grupo externo C. nigrescens polarizando

\begin{tabular}{|c|c|c|c|}
\hline & CP1 & CP2 & CP3 \\
\hline Eigenvalor & 3.5484 & 1.8779 & 1.4684 \\
\hline Poncentaje & 35.4844 & 18.7794 & 14.6836 \\
\hline \%acumilado & 35.4844 & 54.2638 & 68.9474 \\
\hline \multicolumn{4}{|l|}{ Calficaciones } \\
\hline Conteo pectinal & 0.02566 & 0.67022 & -0.04094 \\
\hline Long. totah Long. metasoma & -0.3591 & 0.22453 & 0.2881 \\
\hline Long. metahong cefalotórax & 0.47598 & -0.20543 & -0.04413 \\
\hline $\begin{array}{l}\text { Long. cefalctórax/Long. segmento } \\
\text { caudal V }\end{array}$ & -0.43843 & 0.12529 & 0.11624 \\
\hline Long/Ancho patela & 0.22024 & 0.53424 & -0.03189 \\
\hline Long. /Ancho vesicula & 0.04836 & -0.05336 & -0.6921 \\
\hline Lonḡiud agríón & 0.40582 & 0.27437 & 0.08429 \\
\hline $\begin{array}{l}\text { Dientes accesorios externos del dedo } \\
\text { fjo }\end{array}$ & 0.23579 & 0.04543 & 0.44861 \\
\hline Tubénculo subaculear & 0.27649 & -0.23932 & $\mathbf{0 . 4 3 6 8 3}$ \\
\hline Long. dedo móvill Long. pateła & -0.32253 & -0.14005 & 0.1375 \\
\hline
\end{tabular}

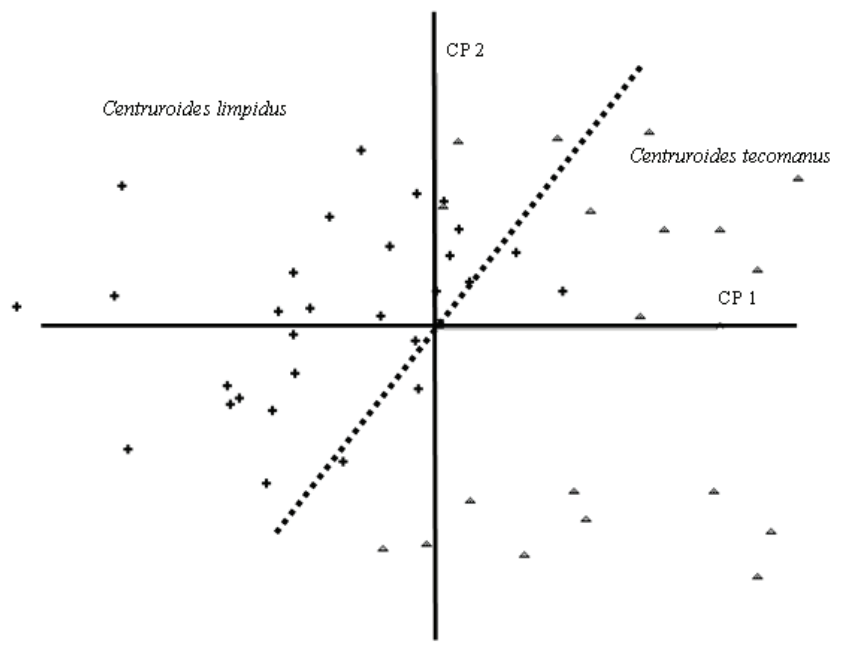

Figura 5. Gráfico de ordenación con ACP y la tabla correspondiente para 10 variables de machos provenientes de poblaciones identificadas como subespecies de Centruroides limpidus, separadas por la línea punteada. En la tabla se resaltan las calificaciones que permiten interpretar el gráfico. Las características con negritas en CP1 deben considerarse diagnósticas.

el cladograma y el otro grupo externo (C. exilicauda) ubicándose entre el clado de "infamatus-tecomanus" y el de los "limpidus limpidus", formando un grupo que se separa del grupo externo C. nigrescens. En los cladogramas generados se aprecia monofilia para los $C$. limpidus, $C$. balsasensis y $C$. infamatus, con altos valores de soporte para cada uno de los clados de interés en este trabajo (Fig. 6 y 7$)$.

La congruencia de los gráficos que se obtuvieron con los métodos utilizados robustece la propuesta de que las poblaciones representadas corresponden a taxones genéticamente distintos en un nivel suficiente para justificar su existencia como especies propias. 


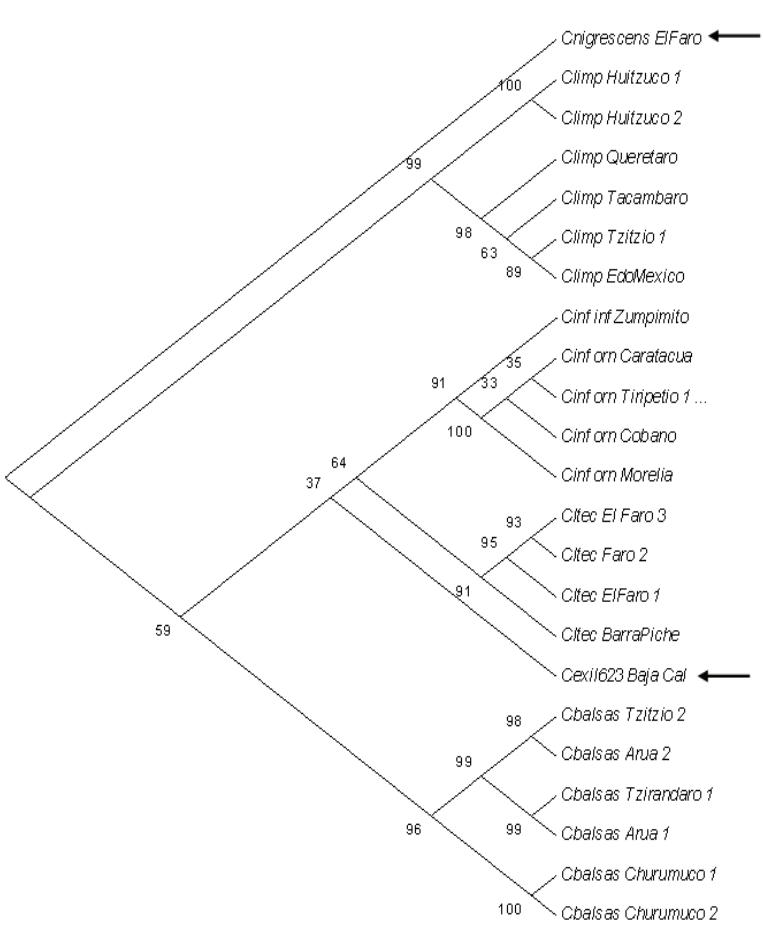

Figura 6. Cladograma obtenido con el método de reconstrucción filogenética del "vecino más cercano" (NJ) para las 3 especies de Centruroides utilizadas en el análisis de secuencias del fragmento de gen mitocondrial 16S. Las flechas indican la posición de los grupos externos. Los cladogramas fueron "enraizados" con $C$. nigrescens. Cnigrescens = Centruroides nigrescens (grupo externo), Cexil $=$ Centruroides exilicauda (grupo externo), Climp $=$ Centruroides limpidus limpidus . Cltec $=$ Centruroides limpidus tecomanus = Centruroides tecomanus stat. nov., Cinf inf $=$ Centruroides infamatus infamatus, Cinf orn $=$ Centruroides infamatus ornatus, Cbalsas = Centruroides balsasensis. Los valores representan la estimación de confiabilidad de los clados y ramas (bootstrapping con 500 réplicas).

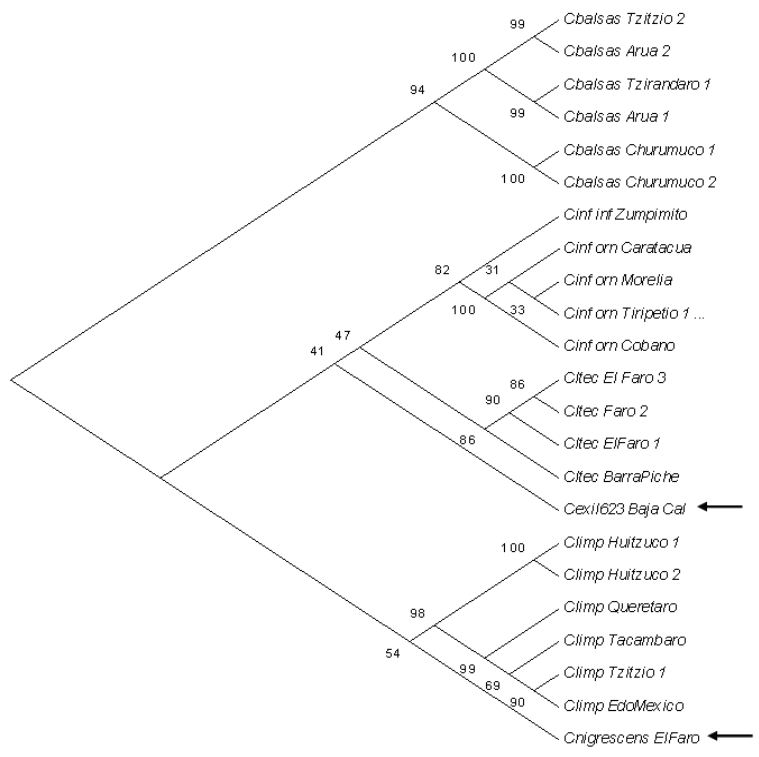

Figura 7. Cladograma obtenido con el método de reconstrucción filogenética de mínima evolución (ME) para las 3 especies de Centruroides utilizadas en el análisis de secuencias del fragmento de gen mitocondrial 16S. Las flechas indican la posición de los grupos externos. Los cladogramas fueron "enraizados" con $C$. nigrescens. Cnigrescens $=$ Centruroides nigrescens (grupo externo), Cexil = Centruroides exilicauda (grupo externo), Climp $=$ Centruroides limpidus limpidus. Cltec $=$ Centruroides limpidus tecomanus $=$ Centruroides tecomanus stat. nov., Cinf inf $=$ Centruroides infamatus infamatus, Cinf orn $=$ Centruroides infamatus ornatus, Cbalsas = Centruroides balsasensis. Los valores representan la estimación de la confiabilidad de los clados y ramas (bootstrapping con 500 réplicas).
Figura 8. Vesícula, aguijón y tubérculo subaculear de (a) C. tecomanus Hoffmann y (b) C. limpidus Karsch.
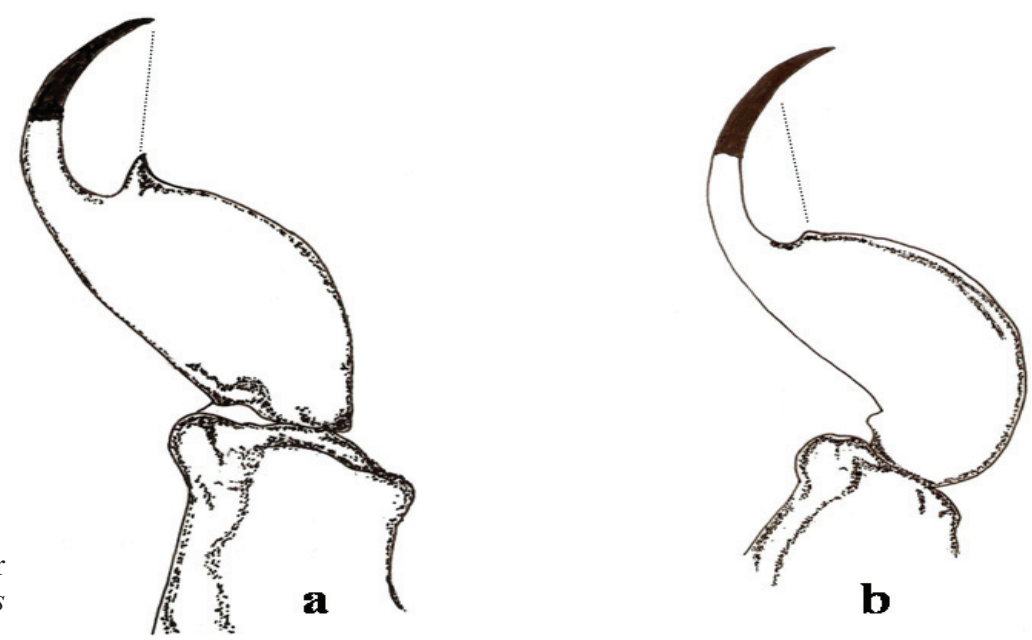


\section{Discusión}

En la primera agrupación obtenida utilizando las 39 variables iniciales, sólo el grupo de los C. balsasensis se mantuvo, tanto en hembras como en machos, mientras que los ejemplares de $C$. limpidus limpidus y C. limpidus tecomanus, tuvieron alto grado de confusión, debido a que muchas de estas características se referían al tamaño y las especies en general son parecidas en esos valores; además de que el conteo pectinal y algunas proporciones también se comparten, generando mezclas por efecto del grado de semejanza morfométrica. La depuración de matriz eliminando variables redundantes (aportan igual información por estar altamente correlacionadas) y ruidosas (confunden las agrupaciones por ser de respuesta discordante al interior de los grupos), permitió una agrupación más clara y la definición de las características útiles para separar los 3 taxones involucrados en el análisis.

La clasificación que se obtuvo con las variables seleccionadas (Cuadro 4) es buena, con sólo un ejemplar macho de $C$. limpidus tecomanus mal clasificado, ya que se confunde con $C$. balsasensis por efecto de la longitud del metasoma, respecto a la del caparazón cefalotorácico y principalmente por la proporción de longitud y ancho de la vesícula, además del conteo de dientes accesorios externos del dedo fijo. El haber obtenido agrupaciones similares al utilizar las variables seleccionadas, independientemente del método de agrupación que se utilice, significa que hay buena compactación y "naturalidad", en el sentido de la taxonomía numérica, en los grupos formados. Las diferencias morfométricas detectadas pueden utilizarse para completar las diferencias morfológicas previamente mencionadas para los 3 taxones en estudio por Ponce y Francke (2004).

Las poblaciones reconocidas inicialmente como C. limpidus limpidus, morfométricamente presentan diferencias importantes con las poblaciones de $C$. limpidus tecomanus y la distribución geográfica conocida para ambas subespecies les ubica en áreas separadas, ya que $C$. limpidus tecomanus es estrictamente costera mientras que las poblaciones de $C$. limpidus limpidus son continentales y aparentemente no cruzan la sierra Madre del Sur, que en el norte de Michoacán y sur de Colima está representada por la sierra de Coalcomán.

De los resultados del análisis morfométrico se pueden establecer características diagnósticas: los machos de $C$. limpidus limpidus tienen proporcionalmente un metasoma más corto respecto a la longitud total y la longitud del caparazón cefalotorácico, lo que se refleja en una proporción mayor entre longitud de metasoma / longitud del caparazón cefalotorácico y una menor proporción, si se relaciona con la longitud total (sin incluir vesícula) respecto a los valores que se obtienen en los ejemplares de C. limpidus tecomanus; el segmento caudal $\mathrm{V}$ es de menor longitud y en la proporción con respecto a la longitud del cefalotórax tendrá valores mayores en relación con la misma proporción en C. limpidus tecomanus. La longitud del dedo móvil es ligeramente mayor que la longitud de la patela en C. limpidus limpidus, reflejada en los valores de la proporción longitud del dedo móvil / longitud de la patela, que son superiores a 1.0 ; mientras que en $C$. limpidus tecomanus tienden a ser semejantes y por tanto la proporción es muy cercana a 1.0 (Cuadro 4).

Una medida directa, claramente distinta, es la longitud del aguijón, el cual evidentemente es más pequeño en $C$. limpidus limpidus $(2.81 \pm 0.27 \mathrm{~mm})$, lo que junto con la característica diagnóstica de Hoffmann (1932) acerca del tubérculo subaculear pobremente desarrollado y granular, son una forma clara de diferenciarle, ya que en C. limpidus tecomanus se encontrará un aguijón más largo (3.14 $\pm 0.25 \mathrm{~mm}$ ) y con el tubérculo subaculear siempre bien desarrollado, cónico, en categorías de grande a muy grande, y con la punta orientada hacia la punta del aguijón (Fig. 8). En las hembras, las proporciones del metasoma respecto a la longitud total y la longitud del caparazón cefalotorácico se mantienen, lo mismo que la relación entre la longitud del cefalotórax y el segmento caudal V. También se observaron diferencias estadísticas en la longitud del dedo fijo, claramente mayor en C. limpidus tecomanus, taxón en el que los segmentos caudales III y IV son más anchos (Cuadro 4). El tubérculo subaculear, morfológicamente distingue a $C$. limpidus de $C$. tecomanus, aunque cuando se analiza de manera multivariada no es una característica determinante (Figs. 4 y 5), por lo que para asegurar la determinación específica se recomienda revisar también las características morfométricas antes mencionadas.

Para este caso, algunas características que generalmente ayudan a la identificación como el conteo pectinal, o la proporción entre la longitud y ancho de la vesícula no fueron útiles, ya que tanto en hembras como en machos $(n=49)$ no se detectaron diferencias estadísticamente significativas $(\mathrm{p}>0.05)$. En el caso de los machos, la proporción entre longitud y ancho de la patela tampoco tuvo diferencia entre estos taxones; pero es importante hacer notar que son características valiosas para diferenciar C. balsasensis, taxón con el que tanto C. limpidus limpidus como C. limpidus tecomanus presentan algunas semejanzas morfométricas (Ponce y Francke, 2004).

Genéticamente, se puede apreciar que $C$. nigrescens, representante de los Centruroides "no rayados", tiene diferencia genética suficiente para mantenerse separado del resto de taxones analizados y el otro grupo externo $C$. exilicauda ubicándose en medio del clado de los "limpidus- 
limpidus" y los "infamatus-tecomanus", justificando la separación propuesta por Hoffmann (1932) entre alacranes "no rayados" y "rayados". En relación con los taxones de interés en este trabajo, de los árboles obtenidos se puede concluir que hay 3 clados diferentes, 1 que es representante del taxón con poblaciones identificadas como C. limpidus limpidus en localidades de los estados de Guerrero, Querétaro, Estado de México y Michoacán; otro, conformado por las poblaciones de C. limpidus tecomanus, con poblaciones en la costa de Colima y Michoacán, desde el norte de este estado hasta la zona de Lázaro Cárdenas, en el límite con Guerrero, donde el río Balsas representa un límite para su distribución, y un tercer clado representado por las poblaciones de $C$. balsasensis con poblaciones mayoritariamente ubicadas en la depresión del Balsas.

Es importante hacer notar que en este análisis, el clado de los C. limpidus tecomanus es hermano del clado formado por $C$. infamatus infamatus y $C$. infamatus ornatus y está claramente separado del clado formado por las poblaciones de C. limpidus limpidus y C. balsasensis representadas en el análisis, lo que corrobora la falta de monofilia para el hasta ahora conocido como grupo "limpidus-elegans", ya detectada por Towler et al. (2001) y Ponce (2003).

De acuerdo con estos resultados, $C$. limpidus tecomanus presenta diferencias morfológicas y genéticas que le separan de $C$. limpidus limpidus y genéticamente le acercan a las poblaciones de $C$. infamatus, a pesar de compartir la característica de coloración de las líneas bien definidas sobre el caparazón cefalotorácico que distingue al grupo "limpidus-elegans"; adicionalmente, al no existir monofilia con los especimenes de C. limpidus limpidus, no hay congruencia con el concepto filogenético de especie y en consecuencia la categoría subespecífica, no es apropiada en su aplicación; por tanto, debe eliminarse para darle el rango de especie propia bajo el nombre de Centruroides tecomanus Hoffmann, 1932 stat. nov. y sugerir la revisión de la propuesta de González-Santillán (2001) sobre el grupo de alacranes rayados "limpidus-elegans", basándose en la presencia de las 4 rayas bien definidas en el caparazón cefalotorácico; ya que las evidencias obtenidas con esta especie la acercan al grupo "suffusus-infamatus" $\mathrm{y}$, al menos genéticamente, no parece ser parte del grupo "limpidus-elegans".

Los resultados de este trabajo también resaltan la necesidad de probar el nivel de divergencia entre las poblaciones analizadas y algunas poblaciones de $C$. elegans (Thorell 1876) para probar la validez del grupo. Respecto a las subespecies de C. infamatus cuyas diferencias genéticas también pueden estar reflejando diferencias morfológicas y de distribución geográfica, los cladogramas obtenidos justifican la necesidad de un análisis sobre el estatus taxonómico actual, como ya lo apuntaban los trabajos de Miranda (2001), Miranda et al. (2001) y Ponce (2003). Como resultado más relevante de este trabajo se propone que la subespecie $C$. limpidus tecomanus se reconozca como especie válida, con las consecuencias nomenclaturales correspondientes.

\section{Agradecimientos}

A la Coordinación de la Investigación Científica de la Universidad Michoacana de San Nicolás de Hidalgo, por el apoyo económico recibido para el trabajo de campo de este proyecto. Al M.C. Edmundo González Santillán, por haber proporcionado ejemplares de Arcelia, Guerrero y de Los Plátanos, Estado de México, para el análisis morfométrico y genético, y a la Biol. Abigail Jaimes, por la elaboración de los mapas de distribución actual de los taxones en estudio. Dos revisores externos nos ayudaron a mejorar todos los aspectos de este trabajo.

\section{Literatura citada}

Beutelspacher, B. C. R. 2000. Catálogo de los alacranes de México. Universidad Michoacana de San Nicolás de Hidalgo, Morelia, Michoacán, México. 175 p.

Brown, C. A. 2001. Allometry of offspring size and number in scorpions. In Scorpions 2001. In memoriam Gary A. Polis, V. Fet y P.A. Selden (eds.) British Arachnological Society, Burnham Beeches. p 307-315.

Brown, C. A., J. M. Davis, D. J. O'Connell y D. R. Formanowitz, Jr. 2002. Surface density and nocturnal activity in a west Texas assemblage of scorpions. Southwestern Naturalist 47:409-419.

Contreras-Garduño J., A. V. Peretti y A. Córdoba A. 2006. Evidence that mating plug is related to null female mating activity in the scorpion Vaejovis punctatus. Ethology 112: $152-163$.

Córdova-Athanasiadis, M. 2005. Escorpiofauna (Arachnida: Scorpiones) del Estado de Morelos, México. Tesis, Facultad de Ciencias Biológicas, Universidad Autónoma del Estado de Morelos, Cuernavaca, Morelos. 109 p.

De Armas, L. F. y E. Martín-Frías. 1998. Presencia del género Tityopsis en México y descripción de una especie nueva (Scorpiones: Buthidae). Anales de la Escuela Nacional de Ciencias Biológicas, Instituto Politécnico Nacional 43:4549.

De Armas, L. F., E. Martín-Frías y J. Estévez-Ramírez. 2003. Lista anotada de las especies mexicanas del género Centruroides Marx, 1890 (Scorpiones, Buthidae). Revista Ibérica de Aracnología 8:93-98.

Fet, V., W. D. Sissom, G. Lowe y M. E. Braunwalder. 2000. Catalog of the scorpions of the world (1758-1998). New York Entomological Society, New York. 690 p.

Francke, O. F. y W. D. Sissom (en prensa). Listas de especies mexicanas., Parte A (disco compacto), Las fuentes del 
conocimiento de la biodiversidad en México, ). J.Soberón, G. Halffter y J. Llorente-Bousquets (coords.). K. J. Sarukhán (comp.gral.) /Segundo Estudio de País./ CONABIO, México, D. F.

Gantenbein, B., V. Fet, C. R. Largiadèr y A. Scholl. 1999. First DNA phylogeny of Euscorpius Thorell, 1876 (Scorpiones: Euscorpiidae) and its bearing on taxonomy and biogeography of this genus. Biogeographica 75:49-65.

Gantenbein, B., V. Fet, M. Barker y A. Scholl. 2000. Nuclear and mitochondrial markers reveal the existence of two parapatric scorpion species in the Alps: Euscorpius germanus (C. L. Koch, 1837) and E. alpha Caporiacco, 1950, stat. nov. (Scorpiones, Euscorpiidae). Revue Suisse Zoologie 107: 843-869.

Gantenbein, B., V. Fet y M. D. Barker. 2001. Mitochondrial DNA reveals a deep, divergent phylogeny in Centruroides exilicauda (Wood, 1863) (Scorpiones, Buthidae). In Scorpions 2001. In Memoriam Gary A. Polis, V. Fet y P. A. Selden (eds.). British Arachnological Society, Burnham Beeches. p. 235-244

Gauch, H. G. 1982. Multivariate Analysis in Community Ecology. Cambridge University Press, Cambridge. 298 p.

González-Santillán, E. 2001. Catálogo de escorpiones de la Colección Nacional de Arácnidos (CNAN). Tesis, Facultad de Ciencias, Universidad Nacional Autónoma de México, México, D. F. 148 p.

González-Santillán, E. s. f. Escorpiones de México. http:www. venenonemia.org, consultada el 6.VII.2007.

Gotelli, N. J. y A. M. Ellison. 2004. A primer of ecological statistics. Sinauer, Sunderland, Massachusetts. 510 p.

Hernández, C. E. 2002. Análisis morfométrico de ocho poblaciones de Centruroides limpidus (Karsch) y sus subespecies mediante el uso de técnicas multivariadas. Tesis, Universidad Michoacana de San Nicolás de Hidalgo. Morelia, Michoacán, México. 86 p.

Hoffmann, C. C. 1932. Monografías para la entomología médica de México. Monografía. 2. Los escorpiones de México (segunda parte) Buthidae. Anales del Instituto de Biología, Universidad Nacional Autónoma de México, Serie Zoología 3:243-361.

Jaimes, A. 2007. Distribución real y potencial de los escorpiones de importancia médica de México. Tesis, Facultad de Ciencias Biológicas, Universidad Autónoma del Estado de Morelos, Cuernavaca, Morelos. 54 p.

Jeannmougin, F., J. D. Thompson, M. Gouy, D. G. Higgins y T. J. Gibson. 1998. Multiple sequence alignment with Clustal X. Trends Biochemical Science 23:403-405.

Kovach Computing Services. 1998. MVSP (Multi-Variate Statistical Package) v. 3.01. Software.

Kumar, S., K. Tamura y M. Nei. 2004. MEGA3: Integrated software for molecular evolutionary genetics analysis and sequence alignment. Briefings in Bioinformatics 5:150-163.

Llorente-Bousquets, J., E. González S., A. N. García A. y C. Cordero. 1996. Breve Panorama de la taxonomía de artrópodos en México. In Biodiversidad, taxonomía y biogeografía de artrópodos de México: hacia una síntesis de su conocimiento, vol. I, J. E. Llorente-Bousquets, A. N. García A. y E. Gonzáles S. (eds.) Comision Nacional para el Conocimiento y Uso de la Biodiversidad / Universidad Nacional Autónoma de México, México, D. F. p. 3-14.

Lourenço, W. R. y W. D. Sissom. 2000. Scorpiones. En Biodiversidad, taxonomía y biogeografía de artrópodos de México, vol. 2. J. E. Llorente-Bousquets, E. GonzálezSoriano y N. Papavero (eds.) Comisión Nacional para el Conocimiento y Uso de la Biodiversidad / Universidad Nacional Autónoma de México, México, D, F, p. 115-135.

Magurran, A. E. 1988. Ecological diversity and its measurement. Princeton University Press., New Jersey. 179 p.

Manly, B. F. J. 1998. Multivariate statistical methods. A primer, 2nd. ed. Chapman \& Hall, London. 215 p.

Miranda, L. E. P. 2001. Utilización de técnicas moleculares para la definición del estatus taxonómico de 2 especies y subespecies del género Centruroides Marx, 1890 (Scorpiones: Buthidae). Tesis, Universidad Michoacana de San Nicolás de Hidalgo. Morelia, Michoacán. 71 p.

Miranda, L. E. P., J. Ponce S. y H. Cano C. 2001. Evidencias morfométricas y genéticas de la existencia de dos linajes específicos en poblaciones reconocidas como Centruroides infamatus (C. L. Koch) y sus taxa subespecíficos (Scorpiones, Buthidae). Memorias del XXXVI Congreso Nacional de Entomología. Sociedad Mexicana de Entomología, Querétaro, Querétaro. p. E32.

Moreno, B. R. J., J. Ponce S. y C. R. Beutelspacher B. 1998. Alacranes (Arachnida: Scorpiones) de cuatro localidades de la zona de transición a la tierra caliente del estado de Michoacán. Memorias del XXXIII Congreso Nacional de Entomología. Sociedad Mexicana de Entomología, Acapulco, Guerrero. p. 440-444.

Nei, M. y S. Kumar. 2000. Molecular Evolution and Phylogenetics. Oxford University Press, New York. 333 p.

Polis, G. A. 1990. Ecology. In The biology of scorpions, G. A. Polis (ed.) Stanford University Press, California. p. 247293.

Polis, G. A. y W. D. Sissom. 1990. Life history. In The biology of scorpions, G. A. Polis (ed.). Stanford University Press, California. p. 161-223.

Ponce, S. J., 2003. Ecología y distribución del género Centruroides Marx 1890 (Scorpiones: Buthidae), en la depresión del Balsas del estado de Michoacán. Tesis, doctorado Facultad de Ciencias Naturales, Universidad Autónoma de Querétaro, Santiago de Querétaro. 276 p.

Ponce, S. J. y C. R. Beutelspacher B. 2001. Alacranes de Michoacán. Ediciones Michoacanas / Universidad Michoacana de San Nicolás de Hidalgo, Morelia, Michoacán. $112 \mathrm{p}$.

Ponce, S. J. y O. F. Francke. 2004. Una nueva especie de alacrán del género Centruroides Marx (1890) (Scorpiones: Buthidae) de la depresión del Balsas, México. ActaZoológica Mexicana, nueva serie 20:221-232.

Ponce, S. J. y R. J. Moreno B. 2003. Alacranes de la depresión del Balsas en el estado de Michoacán. In Entomología mexicana, vol. 2, N. J. Romero, E. G. Estrada V. y A. Equihua M. (eds.). Sociedad Mexicana de Entomología, México, D. F. p. 30-35.

Ponce, S. J. y R. J. Moreno B. 2005. El género Centruroides Marx 1890 (Scorpiones: Buthidae) en México. Biológicas, Revista 
de la Facultad de Biología de la Universidad Michoacana de San Nicolás de Hidalgo 7: 42-51.

Ponce S. J., R. J. Moreno B. y E. P. Miranda L. 2003. Cortejo y comportamiento de apareamiento de Centruroides sp. nov. (Scorpiones: Buthidae) en condiciones de laboratorio. Biológicas, Revista de la Facultad de Biología de la Universidad Michoacana de San Nicolás de Hidalgo 5: 6470.

Rein, J. O. 2006. The scorpion files. Norwegian University of Science and Technology, http.//www.ub.ntnu.no/scorpionfiles/; consultada: julio 2008.

SAS Institute. 2005. JMP Statistical Discovery. versión 6.0.0. Software.
Simpson, J. 1997. Molecular markers. Boletín de la Sociedad Botánica de México 60:73-76.

Towler, W. I., J. Ponce S., B. Gantenbein y V. Fet. 2001. Mitochondrial DNA reveals a divergent phylogeny in tropical Centruroides (Scorpiones: Buthidae) from Mexico. Biogeographica 77:157-172.

Vidal-Acosta, V. y O. F. Francke. (en prensa). Redescripción de Tityopsis aliciae Armas y Martín-Frías (Scorpiones: Buthidae). Revista Mexicana de Biodiversidad.

Yamashita, T. 2004. Surface activity, biomass, and phenology of striped scorpion, Centruroides vittatus (Buthidae) in Arkansas. Euscorpius-Occasional Publications in Scorpiology 17:25-33. 